hep-ph/9812479

DFTT $74 / 98$

INFN-RM3 98/9

\title{
Truncated moments of parton distributions
}

\author{
Stefano Forte円 \\ INFN, Sezione di Roma III \\ Via della Vasca Navale 84, I-00146, Roma, Italy \\ Lorenzo Magnea \\ Dipartimento di Fisica Teorica, Università di Torino \\ and INFN, Sezione di Torino \\ Via P. Giuria 1, I-10125 Torino, Italy
}

\begin{abstract}
We derive evolution equations satisfied by moments of parton distributions when the integration over the Bjorken variable is restricted to a subset $\left(x_{0} \leq\right.$ $x \leq 1$ ) of the allowed kinematical range $0 \leq x \leq 1$. The corresponding anomalous dimensions turn out to be given by a triangular matrix which couples the $N$-th truncated moment with all $(N+K)$-th truncated moments with integer $K \geq 0$. We show that the series of couplings to higher moments is convergent and can be truncated to low orders while retaining excellent accuracy. We give an example of application to the determination of $\alpha_{s}$ from scaling violations.
\end{abstract}

December, 1998

\footnotetext{
${ }^{1}$ On leave from INFN, Sezione di Torino, Italy
} 
The description of scaling violations of deep-inelastic structure functions is historically one of the first predictions of perturbative QCD amenable to experimental testing, and still is one of the most accurate ways to use perturbative QCD for precision measurements [1]. Specifically, comparison of the theoretical prediction with the data allows a determination of the only free parameter in the QCD lagrangian, the strong coupling $\alpha_{s}$, as well as the extraction of the parton distributions of hadrons which, though in principle computable, are determined from the nonperturbative dynamics of the theory and thus must be treated as unknown phenomenological parameters.

As well known, scaling violations are described by ordinary linear differential equations for evolution in $t \equiv \ln \frac{Q^{2}}{\Lambda^{2}}$ satisfied by the Mellin moments of parton distributions, which can be directly viewed as matrix elements of local operators. At the leading log level, parton distributions can be expressed directly as linear combinations of measurable structure functions; beyond leading log this is only true in specific factorization schemes, whereas in a general scheme the moments of structure functions are related to moments of parton distributions through Wilson coefficients which are calculable as perturbative expansions in $\alpha_{s}$ [2].

From a phenomenological point of view, however, dealing with moments of structure functions is rather inconvenient, since by definition Mellin moments are obtained integrating over all values of the Bjorken variable $0 \leq x \leq 1$. Since $x$ is related to the invariant energy $W^{2}$ of the virtual photon-hadron scattering process by $W^{2}=\frac{1-x}{x}, x \rightarrow 0$ is the infinite energy limit and can thus never be attained experimentally. All moments are thus subject to an a priori infinite uncertainty from this region, which can only be reduced on the basis of nonperturbative models and assumptions, such as the idea that the virtual photon-hadron scattering cross section should behave at high energy like the cross section for scattering between real hadrons and thus be controlled by Regge theory [3]. It follows that any use of the evolution equations for moments requires some model-dependent input.

A way to avoid this problem is of course well known: work in $x$-space and deal with Altarelli-Parisi equations, which give directly the evolution of parton distributions (rather than of their moments). Undoing the moments turns the ordinary differential equations in $t$ into integro-differential equations in $x$ and $t$, but the $x$ integration is such that scaling violations at $x_{0}$ only depend on the values of parton distributions for all $x \geq x_{0}$. The need for an extrapolation to the unmeasurable $x \rightarrow 0$ region is thus at least in principle avoided [4].

In practice, however, in order to be able to solve the Altarelli-Parisi equation, we must parameterize the data in some specific way. This is usually done by fitting a well-defined functional form $f(x)$, parametrized by some free parameters, to data at a fixed scale $Q^{2}$; or, alternatively, expanding on suitable basis of functions [5]. In either case, a bias is introduced in the analysis, due to the fact that the specific choice of functional form of the fitting function, or of the basis functions, constrains, for obvious reasons of smoothness, the description of data with the smallest measured values of $x$. Giving a precise quantitative estimate of the possible bias thus 
introduced can in practice be rather complicated [6].

One may also be interested in using scaling violations for the determination of a moment of a parton distribution. For instance, the gluon distribution is primarily determined from scaling violations [7], and one may be interested directly in its moments because of their physical interpretation, or because the structure of evolution equations is such that some moments are better constrained by an observed pattern of scaling violations than the parton distribution for any individual value of $x$ [8]. However, when arriving at a determination of, say, the first moment of the polarized gluon distribution [6], it would be very useful to be able to separate the contribution to the moment from the measured region - which is determined from the data up to conventional uncertainties - from that due to an extrapolation which is necessarily entirely based on theoretical prejudice.

All these problems are solved if one is able to formulate evolution equations directly in terms of moments restricted to the measured region. Since, as already mentioned, evolution at $x_{0}$ requires knowledge of the parton distribution for all $x \geq x_{0}$, it is clear that the scale dependence of a moment evaluated by integrating over the restricted range $x_{0} \leq x \leq 1$ (truncated moment, henceforth) rather than over the full kinematically allowed region $0 \leq x \leq 1$, is determined by the structure function in the same region. [ It is however not obvious that the evolution equations for truncated moments will take a simple form, or even that they will close upon a specific subset of moments: if they did not, nothing would be gained by introducing truncated moments over the resolution of the full Altarelli-Parisi equations.

Here, we will derive evolution equations for truncated moments. We will show that the evolution of truncated moments is driven by a triangular matrix of anomalous dimensions which couples the $N$-th truncated moment to all $N+K$-th moments, where $K$ is a positive integer (but $N$ can be any real number). The elements of this matrix of anomalous dimensions depend on the cutoff $x_{0}$, and are calculable in perturbation theory as straightforward integrals of the Altarelli-Parisi splitting functions. Furthermore, we will prove that the series of couplings to higher moments is convergent, so that the infinite matrix of anomalous dimensions can be truncated to specified accuracy. We will also see that this convergence is very fast, so in practice it is only necessary to deal with small (typically less than $10 \times 10$ ) matrices.

In order to derive the evolution equations for truncated moments, we start from

\footnotetext{
${ }^{2}$ Turning the argument around, this also means that evolution equations for moments truncated to a generic interval $x_{0} \leq x \leq x_{1}$ do not close, because they would also require knowledge of parton distributions for $x>x_{1}$. Hence, problems related to the extrapolation to $x=1$ of data taken only at $x \leq x_{1}$ cannot be solved by the methods of this paper. The extrapolation to $x=1$ is however a much less serious problem, both because the $x \rightarrow 1$ limit is, unlike the $x \rightarrow 0$ limit, at least in principle experimentally accessible, and because structure functions must vanish as $x \rightarrow 1$ for kinematic reasons, so that the extrapolation is under much better control.
} 
the Altarelli-Parisi evolution equation

$$
\frac{d}{d t} q\left(x, Q^{2}\right)=\frac{\alpha_{s}\left(Q^{2}\right)}{2 \pi} \int_{x}^{1} \frac{d y}{y} P\left(\frac{x}{y} ; \alpha_{s}\left(Q^{2}\right)\right) q\left(y, Q^{2}\right) .
$$

Here $q\left(x, Q^{2}\right)$ is the nonsinglet quark distribution; in the singlet case one must consider a $2 \times 2$ matrix of anomalous dimensions which mixes the quark and gluon distributions. This introduces some trivial complications which we will not discuss here. The splitting function $P(x)$ is given as a series in $\alpha_{s}, P(x)=P^{(0)}+\frac{\alpha_{s}}{2 \pi} P^{(1)}+\ldots$; henceforth we will suppress its explicit $\alpha_{s}$ dependence.

The truncated Mellin moment of the parton distribution $q\left(x, Q^{2}\right)$ is defined as

$$
q_{N}\left(x_{0}, Q^{2}\right) \equiv \int_{x_{0}}^{1} d x x^{N-1} q\left(x, Q^{2}\right) .
$$

By integrating Eq. (1) and inverting the order of the double integration it is easy to see that truncated moments satisfy the evolution equation

$$
\frac{d}{d t} q_{N}\left(x_{0}, Q^{2}\right)=\frac{\alpha_{s}\left(Q^{2}\right)}{2 \pi} \int_{x_{0}}^{1} d y y^{N-1} q\left(y, Q^{2}\right) G_{N}\left(\frac{x_{0}}{y} ; \alpha_{s}\left(Q^{2}\right)\right),
$$

with an evolution kernel $G_{N}$ given by a truncated moment of the splitting function $P(z)$,

$$
G_{N}(x)=\int_{x}^{1} d z z^{N-1} P(z) .
$$

If $x_{0}=0$ the kernel $G_{N}\left(x_{0} / y\right)$ reduces to the usual $x$-independent anomalous dimension, $G_{N}(0)=\gamma_{N}$, it can thus be taken outside the integral in Eq. (3), and the r.h.s. of Eq. (3) depends only on the $N$-th moment. Henceforth we will assume that $\boldsymbol{R e} N$ is large enough for $\gamma_{N}$ to be regular. If instead $x_{0} \neq 0$, because of the residual $y$ dependence in the kernel $G_{N}$ the evolution equation does not diagonalize. However, it is easy to see that the $N$-th truncated moment mixes only with moments with index $M \geq N$.

To prove this, expand the kernel $G_{N}$ in Taylor series around $y=1$.

$$
G_{N}\left(\frac{x_{0}}{y}\right)=\sum_{n=0}^{\infty} \frac{g_{n}^{N}\left(x_{0}\right)}{n !}(y-1)^{n},
$$

where

$$
\left.g_{n}^{N}\left(x_{0}\right) \equiv \frac{\partial^{n}}{\partial y^{n}} G_{N}\left(\frac{x_{0}}{y}\right)\right|_{y=1} .
$$

Since $G_{N}(z)$ is regular for all $0 \leq z<1$, but in general has logarithmic singularities as $z \rightarrow 1$, due to the presence of + distributions in the splitting function $P(z)$, the Taylor expansion in Eq. (5) has radius of convergence $r=\left(1-x_{0}\right)$. However, since the singularities of $G_{N}\left(x_{0} / y\right)$ at $y=x_{0}$ are integrable, we can substitute the Taylor expansion Eq. (5) in the r.h.s. of the evolution Eq. (3), exchange the order 
of sum and integral, and still end up with a convergent sum. Since $G_{N}\left(x_{0} / y\right)$ is regular at $y=1$, the expansion Eq. (5) contains only non-negative powers of $y$, so, after substitution of the expansion in Eq. (3), there is no mixing between the $N$-th truncated moment and moments with $M<N$, as promised.

Because of the convergence of the Taylor expansion (5) and of the ensuing expansion of the r.h.s. of Eq. (3), we can truncate the expansion at finite order $M$. A straightforward computation then leads to

$$
G_{N}\left(\frac{x_{0}}{y}\right)=\sum_{K=0}^{M} c_{K, N}^{(M)}\left(x_{0}\right) y^{K}+O\left[(y-1)^{M+1}\right]
$$

where

$$
c_{K, N}^{(M)}\left(x_{0}\right)=\sum_{p=K}^{M} \frac{(-1)^{K+p} g_{p}^{N}\left(x_{0}\right)}{K !(p-K) !},
$$

so that the evolution equation (3) becomes

$$
\frac{d}{d t} q_{N}\left(x_{0}, Q^{2}\right)=\frac{\alpha_{s}\left(Q^{2}\right)}{2 \pi} \sum_{K=0}^{M} c_{K, N}^{(M)}\left(x_{0}\right) q_{N+K}\left(x_{0}, Q^{2}\right)
$$

We are thus led to an ordinary finite system of differential equations, which can be solved by standard methods, provided the number of equations in the system equals the number of unknowns. For this to happen, we must include a decreasing number of terms in the series (5) as the order of the moment increases. For example, if one is interested in the evolution of the $N_{0}$-th moment, and wishes to include $M+1$ terms in the series that expresses its scale dependence, one must include in the series associated with the $\left(N_{0}+K\right)$-th moment only $M+1-K$ terms. This then gives an upper triangular matrix of coefficients. Such an approximation is only possible if higher moments have a decreasing influence on the evolution, so they may be approximated less accurately.

It is easy to see that this is indeed the case. If the Taylor expansion Eq. (5) is truncated at order $M$, the percentage error on the r.h.s. of the evolution Eq. (9), due to the truncation of the series, is equal to

$$
R\left(N, M ; x_{0}, Q^{2}\right) \equiv \frac{1}{\mathcal{N}} \int_{x_{0}}^{1} d y y^{N-1} q\left(y, Q^{2}\right)\left[G_{N}\left(\frac{x_{0}}{y}\right)-\sum_{K=0}^{M} c_{K, N}^{(M)}\left(x_{0}\right) y^{K}\right]
$$

where the normalization is given by the exact integral

$$
\mathcal{N}=\int_{x_{0}}^{1} d y y^{N-1} q\left(y, Q^{2}\right) G_{N}\left(\frac{x_{0}}{y}\right) .
$$

Now, the coupling of the $N$-th moment to the $(N+M)$-th moment is due to terms which are at least of order $M$ in the Taylor expansion Eq. (5) (because the expansion of the coefficient $c_{K, N}$ in Eq. (8) starts at order $K$ ). Such terms decrease 
very rapidly because the Taylor series is convergent, and furthermore the relative size of the $(N+M)$-th moment compared to the $N$-th moment decreases rapidly, since parton distributions fall off as a power of $(1-x)$ as $x \rightarrow 1$. We will check this explicitly below in the particular case of the NLO evolution of the nonsinglet quark distribution, however we emphasize that it follows from rather general properties of parton distributions and their evolution.

Once the system (9) has been truncated to a finite size, it is straightforward to solve the evolution equations explicitly, by techniques analogous to those used to solve the standard coupled singlet evolution equations. At leading order, the evolution kernels are $t$-independent, so the only $t$ dependence on the r.h.s. of the evolution equation is in the explicit factor of $\alpha_{s}\left(Q^{2}\right)$. The solution is thus simply obtained by diagonalizing the matrix of coefficients, a task which is in our case is enormously simplified by the fact that the matrix is upper triangular.

Let us derive the leading order solution to the evolution equation of the $N_{0}$-th truncated moment, using Eq. (9), which we can rewrite in simplified notation as

$$
\frac{d q_{K}}{d t}=\frac{\alpha_{s}\left(Q^{2}\right)}{2 \pi} \sum_{L=N_{0}}^{N_{0}+M} C_{K L} q_{L}
$$

where $N_{0} \leq K, L \leq N_{0}+M$, and the matrix of coefficients is given by

$$
\left\{\begin{array}{cccc}
C_{K L} & = & c_{L-K, K}^{\left(M-K+N_{0}\right)}\left(x_{0}\right) & (L \geq K) \\
C_{K L} & = & 0 &
\end{array} \quad, \quad\right.
$$

A few basic properties of triangular matrices are collected in the Appendix. One of them, useful below, is the fact that the eigenvalues of a triangular matrix such as $C$ coincide with the diagonal elements, $C_{K K}=c_{0, K}^{\left(M-K+N_{0}\right)}$.

Define now the rotated moments, in the basis in which $C$ is diagonal

$$
\hat{q}_{K}=\sum_{L=N_{0}}^{N_{0}+M} R_{K L} q_{L}
$$

where

$$
\sum_{L, P=N_{0}}^{N_{0}+M} R_{K L} C_{L P} R_{P Q}^{-1}=C_{K K} \delta_{K Q} .
$$

The matrix $R$ which diagonalizes $C$, and its inverse $R^{-1}$, are also upper triangular, and can both be computed exactly by means of a simple recursion relation in terms of the elements of $C$, without having to resort to the time-consuming evaluation of determinants (see the Appendix). It is apparent that the rotated moments evolve independently, and the solution to their evolution equation is given by the familiar expression

$$
\hat{q}_{K}\left(x_{0}, Q^{2}\right)=\left[\frac{\alpha_{s}\left(Q_{0}^{2}\right)}{\alpha_{s}\left(Q^{2}\right)}\right]^{C_{K K} / b_{0}} \hat{q}_{K}\left(x_{0}, Q_{0}^{2}\right)
$$


where $b_{0}$ is the leading coefficient of the $\beta$ function, $b_{0}=11 / 2-n_{f} / 3$ for $S U(3)$. The solution to the evolution equation for truncated moments is then found by simply rotating back,

$$
q_{K}=\sum_{L=N_{0}}^{N_{0}+M} R_{K L}^{-1} \hat{q}_{L}
$$

At next-to-leading order, the evolution kernel for truncated moments, $G_{N}$, acquires a scale dependence though $\alpha_{s}\left(Q^{2}\right)$, and can be written as

$$
G_{N}\left(\frac{x_{0}}{y}, \alpha_{s}\left(Q^{2}\right)\right)=G_{N}^{(0)}\left(\frac{x_{0}}{y}\right)+\frac{\alpha_{s}\left(Q^{2}\right)}{2 \pi} G_{N}^{(1)}\left(\frac{x_{0}}{y}\right)
$$

The NLO evolution equation can then be written as

$$
\frac{d q_{K}}{d t}=\frac{\alpha_{s}\left(Q^{2}\right)}{2 \pi} \sum_{L=N_{0}}^{N_{0}+M}\left[C_{K L}^{(0)}+\frac{\alpha_{s}\left(Q^{2}\right)}{2 \pi} C_{K L}^{(1)}\right] q_{L}
$$

where the matrices $C^{(0)}$ and $C^{(1)}$ are given by Eq. (13) in terms of the coefficients $c_{K, N}^{(0)}$ and $c_{k, N}^{(1)}$ constructed according to Eqs. (5-8) from the LO and NLO kernels respectively.

Diagonalizing the matrix of leading order anomalous dimensions with the matrix $R$ of Eqs. (14) and (15) we get an evolution equation for the rotated moments $\hat{q}$, of the form

$$
\frac{d \hat{q}_{K}}{d t}=\frac{\alpha_{s}\left(Q^{2}\right)}{2 \pi} \sum_{L=N_{0}}^{N_{0}+M}\left[C_{K K} \delta_{K L}+\frac{\alpha_{s}\left(Q^{2}\right)}{2 \pi} \hat{D}_{K L}\right] \hat{q}_{L}
$$

where

$$
\hat{D}_{K L}=\sum_{P, Q=N_{0}}^{N_{0}+M} R_{K P} C_{P Q}^{(1)} R_{Q L}^{-1} .
$$

The matrix evolution equation (20) can be solved with standard techniques of perturbation theory. The evolved (rotated) moments are expressed in terms of the initial condition and of the various anomalous dimensions involved as

$$
\begin{aligned}
\hat{q}_{K}\left(Q^{2}\right)= & {\left[\frac{\alpha_{s}\left(Q_{0}^{2}\right)}{\alpha_{s}\left(Q^{2}\right)}\right]^{C_{K K} / b_{0}}\left[1-\frac{C_{K K} b_{1}}{2 \pi b_{0}^{2}}\left(\alpha_{s}\left(Q_{0}^{2}\right)-\alpha_{s}\left(Q^{2}\right)\right)\right] \hat{q}_{K}\left(Q_{0}^{2}\right) } \\
- & \sum_{L=N_{0}}^{N_{0}+M} \frac{\hat{D}_{K L}}{2 \pi} \frac{1}{C_{K K}-C_{L L}+b_{0}}\left[\left(\frac{\alpha_{s}\left(Q_{0}^{2}\right)}{\alpha_{s}\left(Q^{2}\right)}\right)^{C_{L L} / b_{0}} \alpha_{s}\left(Q^{2}\right)\right. \\
& \left.-\left(\frac{\alpha_{s}\left(Q_{0}^{2}\right)}{\alpha_{s}\left(Q^{2}\right)}\right)^{C_{K K} / b_{0}} \alpha_{s}\left(Q_{0}^{2}\right)\right] \hat{q}_{L}\left(Q_{0}^{2}\right)
\end{aligned}
$$

where $b_{1}$ is the second coefficient of the QCD $\beta$ function, $b_{1}=51 / 2-19 n_{f} / 6$ for $S U(3)$. The NLO solution is then found once again by just rotating back according to Eq. (17). 
In order to show this formalism at work, we consider now the determination of $\alpha_{s}$ from scaling violations of the nonsinglet structure function $F_{2}^{\mathrm{NS}}$. Our method allows a direct determination of the strong coupling by fitting the evolution of the truncated moments of the measured structure function, with $\alpha_{s}$ left as the only free parameter, without having to introduce a parametrization of parton distributions. A determination of $\alpha_{s}$ from scaling violations of a nonsinglet moment has been sometimes attempted, but only in the presence of sum rules which fix the asymptotic normalization of the moment [6, 10]. However, even the presence of this constraint does not obviate the problem of the uncertainty introduced by the small $x$ extrapolation, which remains sizable [1, 6].

The structure function $F_{2}^{\mathrm{NS}}$ in the DIS scheme [9] is simply equal to the nonsinglet quark distribution,

$$
\begin{aligned}
F_{2}^{\mathrm{NS}}\left(x, Q^{2}\right) & \equiv\left(F_{2}^{\mathrm{p}}\left(x, Q^{2}\right)-F_{2}^{\mathrm{n}}\left(x, Q^{2}\right)\right) \\
& =\left.\sum_{i=1}^{n_{f}} e_{i}^{2}\left[q_{i}\left(x, Q^{2}\right)+\bar{q}_{i}\left(x, Q^{2}\right)\right]\right|_{p-n}
\end{aligned}
$$

In order to determine its evolution it is thus sufficient to transform to the DIS scheme the well-known NLO nonsinglet splitting function $P_{q q}^{\mathrm{NS}}$ [11]. We can then determine the evolution kernel $G_{N}(z)$ Eq. (州) analytically, and study the accuracy of the truncation of the expansion in Eq. (5) by explicitly computing the function $R\left(N, M ; x_{0}, Q^{2}\right)$ defined in Eq. (10). For this purpose, we must use an explicit nonsinglet quark distribution, which we can take from any recent parton distribution set. We then evaluate the function $R$ at the reference scale of the chosen parton set, with several typical choices of the lower limit $x_{0}$ of the $x$-range, by including a decreasing number of terms as the order of the moment increases, as discussed above.

The results are shown in Table 1 for moments between the second and the fifth. It is apparent that, despite the fact that less terms are included, the accuracy of the determination of higher moments is actually higher: the fact that higher moments are largely insensitive to the lower limit of integration overwhelms the error introduced by the truncation of the Taylor expansion in Eq. (5). In particular, it is apparent that in order to reliably compute the evolution of the second moment it suffices to consider a four by four evolution matrix. An accurate description of the scaling violations of the first moment would instead require the inclusion of several more terms; this is a consequence of the fact that the integrand in Eq. (4) decreases as $z \rightarrow 0$ only if $N>1$. The convergence to the correct result is of course slower when $x_{0}$ is larger, since the dependence on $y$ of the kernel Eq. (5) is weaker when $x_{0}$ is smaller (and indeed, there would be none in the limit $x_{0}=0$ ).

The simplest way to determine $\alpha_{s}$ is to extract from the data the required set of moments, for instance the moments from the second to the fifth, as in Tab. 1, at the scale where the kinematic coverage in $x$ is widest at large $x$ (so all moments can be reliably determined), then solve the evolution equation, compare to the data 


\begin{tabular}{|l||l|l|l||l|l|l|}
\hline$x_{0}$ & 0.01 & 0.03 & 0.1 & 0.01 & 0.03 & 0.1 \\
\hline $\mathrm{N}$ & \multicolumn{3}{|c||}{$\mathrm{LO}$} & \multicolumn{3}{|c|}{ NLO } \\
\hline \hline 2 & $6.310^{-3}$ & $3.310^{-2}$ & $1.510^{-1}$ & $3.510^{-3}$ & $2.710^{-2}$ & $2.010^{-1}$ \\
\hline 3 & $1.010^{-4}$ & $1.710^{-3}$ & $3.010^{-2}$ & $6.310^{-5}$ & $2.810^{-3}$ & $3.310^{-2}$ \\
\hline 4 & $1.710^{-6}$ & $8.610^{-5}$ & $5.110^{-3}$ & $1.110^{-6}$ & $6.910^{-5}$ & $5.510^{-3}$ \\
\hline 5 & $2.710^{-8}$ & $4.110^{-6}$ & $8.310^{-4}$ & $1.810^{-8}$ & $3.310^{-6}$ & $8.710^{-4}$ \\
\hline
\end{tabular}

Table 1: The percentage error function $R\left(N, M ; x_{0}, Q^{2}\right)$ defined in Eq. (10), computed from the LO and NLO contributions to the nonsinglet splitting function in the DIS scheme, with $M=5-N$, the values of $N$ and $x_{0}$ shown, $Q^{2}=2.56 \mathrm{GeV}^{2}$ and nonsinglet quark distribution from the CTEQ4D parton set [12].

for the second moment at other scales (where, for example, the coverage at large $x$ might be smaller so higher moments are less accurately determined), and perform a fit of $\alpha_{s}$. Our purpose here is not to perform a detailed phenomenological analisys, but rather to explore the viability of the method.

We have thus simply attempted such a fit of $\alpha_{s}$ by using as "data" a parametrization of all available data on $F_{2}\left(x, Q^{2}\right)$ for proton and deuteron targets [13], which (neglecting nuclear effects) determines the nonsinglet as $F_{2}^{\mathrm{NS}}=2\left(F_{2}^{\mathrm{p}}-F_{2}^{\mathrm{d}}\right)$; the moments are then simply found by numerical integration of the parametrization. The kinematic range is essentially limited by the availability of deuterium data: even with a truncation point $x_{0}=0.1$, a reliable reconstruction of the moments is possible only for $30 \mathrm{GeV}^{2} \lesssim Q^{2} \lesssim 100 \mathrm{GeV}^{2}$. Imposing that power corrections be negligible requires the lower cut at $Q^{2}>30 \mathrm{GeV}^{2}$. In fact, since power corrections are large at large $x$ [1], this is important in order for the determination of the higher moments to be reliable.

With all these cuts, fitting to such "data" gives $\alpha_{s}\left(M_{z}\right)=0.115$. An estimate of the statistical error is unfortunately impossible, since a reliable determination of the covariance matrix for the best-fit parameters is not available [14 for the fits of Ref. [13].] However, the fact that the central value is so close to the current global DIS average [15] $\left[\alpha_{s}\left(M_{z}\right)=0.117 \pm 0.002\right.$ (exp.) $\pm 0.004($ th. $\left.)\right]$ suggests that the statistical error is rather small.

It thus appears worth considering an actual extraction of $\alpha_{s}$ from the data using

\footnotetext{
${ }^{3}$ Since the parametrization of $F_{2}$ [13] is provided with an "estimated error band" one might hope to get a qualitative idea of the error by taking the integrals of the upper and lower curves of the band as estimates of the error on the moment. This procedure is however meaningless, as seen by noting that the error on $\alpha_{s}$ could then be made arbitrarily small by increasing the number of values of $Q^{2}$ at which the moment is evaluated (even within a fixed range in $Q^{2}$ ). This apparently paradoxical result is of course due to the fact that the procedure neglects correlations between the values of the moment extracted from the fit at two different scales, which tend to one as the scales get closer.
} 
this method, either by computing the moments numerically from a single set of data, or by first determining a global parametrization of the data with correlations taken into account as required. Such an extraction could presumably be improved by optimizing the choice of moment to be fitted. Indeed, high moments cannot be determined accurately from the data, due to the poor knowledge of structure functions at large $x$, while the evolution of the first moment (which is the lowest convergent one in the nonsinglet case) is hard to determine accurately due to the slower convergence of the expansion Eq. (5). Note that the optimal moment need not be integer, so one would rather expect to have an optimal range. Determinations of $\alpha_{s}$ from different moments in this range could then be combined by properly taking their correlations into account. The extension of the formalism to the singlet sector (which is essentially straightforward) will allow both a determination of $\alpha_{s}$ from wider data sets, and a determination of the partial moments of the gluon distribution, which, as we already discussed, might be of great phenomenological relevance.

In summary, we have determined the evolution equations for truncated moments of parton distribution, and given their explicit solution to NLO in the nonsinglet sector. From a theoretical viewpoint, this fills an obvious gap in the available abundant literature on QCD evolution equations and the methods for solving them. From a phenomenological viewpoint, our results are a useful addition to the set of tools available to extract information from the data on scaling violations, and in particular provide a new way of dealing with the well-known problem of working around our ignorance of the small- $x$ behavior of structure functions. A preliminary extraction of $\alpha_{s}\left(M_{z}\right)$ from the scaling violations of the nonsinglet second moment looks very promising. While we postpone to future work a fuller phenomenological analysis, we encourage experimental collaborations, which necessarily have a much better control of the experimental systematics, to use the simple technique presented in this paper as a means to present data on the moments of the gluon distribution, as well as to obtain determinations of $\alpha_{s}$, which would be significantly less dependent on model assumptions, in comparison to those obtained with more standard techniques.

\section{Appendix}

We list here a few useful properties of triangular matrices. Consider a generic $n \times n$ upper triangular matrix $T_{n}$, with matrix elements $a_{i j},\left(a_{i j}=0\right.$ for $\left.i>j\right)$. It is straightforward to show that:

a) The matrices $T_{n}$ form a proper subgroup of $G L(n)$. 
b) The eigenvalues of $T_{n}$ coincide with the diagonal entries $a_{j j}$. This can be seen noting that the secular equation receives contributions only from the diagonal, since all other minors of $T_{n}-\lambda \mathbf{1}$ vanish.

c) Let $E_{n}$ be the matrix of right eigenvectors of $T_{n}$, arranged in columns. $E_{n}$ is also an upper triangular matrix, which can be chosen to have all diagonal elements equal to unity. Specifically, let $\beta^{(j)}$ be the $j$-th right eigenvector of $T_{n}$, defined by

$$
T_{n} \beta^{(j)}=a_{j j} \beta^{(j)},
$$

and let $\beta_{i}^{(j)}$ be the $i$-th component of the $j$-th eigenvector. Then one can choose $\beta_{j}^{(j)}=1$, and one sees that $\beta_{i}^{(j)}=0$ for $i>j$. Furthermore, the matrix $E_{n}$, with elements $E_{n, i j}=\beta_{i}^{(j)}$, satisfies

$$
E_{n}^{-1} T_{n} E_{n}=\operatorname{diag}\left(a_{j j}\right)
$$

d) The nonvanishing elements of $E_{n}$ satisfy the recursion relation

$$
\beta_{i}^{(j)}=\frac{1}{a_{j j}-a_{i i}} \sum_{p=i+1}^{j} a_{i p} \beta_{p}^{(j)}
$$

initialized by $\beta_{j}^{(j)}=1$. This recursion relation can actually be solved explicitly in terms of minors of the matrix $T_{n}$, however for our purposes the recursion relation itself is more useful, since it can easily be implemented in an evolution program.

e) The inverse matrix $E_{n}^{-1}$ is also upper triangular with unit diagonal entries, and can be constructed noting that, for a generic nonsingular square matrix, the matrix of right eigenvectors (arranged in columns) is invertible, and the inverse is the matrix of left eigenvectors (arranged in rows). This leads to a recursion relation for the elements of $E_{n}^{-1}$, analogous to Eq. (26). Setting $\left(E_{n}^{-1}\right)_{i j}=\gamma_{i}^{(j)}$ we find

$$
\gamma_{i}^{(j)}=\frac{1}{a_{i i}-a_{j j}} \sum_{p=i}^{j-1} \gamma_{i}^{(p)} a_{p j}
$$

It follows that triangular matrices can be diagonalized without computing any determinants. The implementation of the recursion relations Eqs. (26-27) is in fact so efficient that the entire solution of the evolution equations (for reasonably sized matrices, say $n<7-8$ ) can be performed analytically, for arbitrary $x_{0}$, and the chosen value of $x_{0}$ substituted only at the end. 


\section{Acknowledgements}

We would like to thank M. Caselle, G. d'Agostini and P. Provero for discussions, T. Çuhadar, A. Deshpande and E. Rondio for informations on the fits of ref. [13], and R. Ball for helping us to sharpen the derivation of Eq. (9).

\section{References}

[1] See e.g. S. Forte, hep-ph/9812382 and ref. therein.

[2] See R.K. Ellis, W.J. Stirling and B. Webber, QCD and Collider Physics (Cambridge U.P., Cambridge, U.K., 1996).

[3] See e.g. A. Donnachie and P. V. Landshoff, Z. Phys. C61 (1994) 139.

[4] G. Altarelli and G. Parisi, Nucl. Phys. B126 (1977) 298.

[5] W. Furmański and R. Petronzio, Nucl. Phys. B195 (1982) 237.

[6] G. Altarelli et al, Nucl. Phys. B496, 337 (1997); Acta Phys. Pol. B19 (1998) 1145.

[7] J. Huston et al, Phys. Rev. D58 (1998) 114034.

[8] S. Forte, G. Altarelli and G. Ridolfi, hep-ph/9808462.

[9] G. Altarelli, R. K. Ellis and G. Martinelli, Nucl. Phys. B143 (1978) 521; B157 (1979) 461.

[10] CCFR Coll., J. H. Kim, D. A. Harris et al, Phys. Rev. Lett. 81 (1998) 3595.

[11] G. Curci, W. Furmański and R. Petronzio, Nucl. Phys. B175 (1980) 27.

[12] H. L. Lai et al., Phys. Rev. D55 (1997) 1280.

[13] SMC Coll., B. Adeva et al., Phys. Rev. D58 (1998) 112001; T. Çuhadar, Ph. D. thesis, Amsterdam Free Univeristy, 1998.

[14] T. Çuhadar and E. Rondio, private communication.

[15] Particle Data Group, C. Caso et al., Eur. Phys. J. C3 (1998) 1. 